\title{
Active and Nonlinear Microrheology in Dense Colloidal Suspensions
}

\author{
I. Gazuz, ${ }^{1}$ A. M. Puertas, ${ }^{2}$ Th. Voigtmann, ${ }^{1,3}$ and M. Fuchs ${ }^{1}$ \\ ${ }^{1}$ Fachbereich Physik, Universität Konstanz, 78457 Konstanz, Germany \\ ${ }^{2}$ Departamento de Física Aplicada, Universidad de Almería, 04.120 Almería, Spain \\ ${ }^{3}$ Institut für Materialphysik im Weltraum, Deutsches Zentrum für Luft- und Raumfahrt (DLR), 51170 Köln, Germany
}

(Dated: October 26, 2018)

\begin{abstract}
We present a first-principles theory for the active nonlinear microrheology of colloidal model systems: for constant external force on a spherical probe particle embedded in a dense host dispersion, neglecting hydrodynamic interactions, we derive an exact expression for the friction. Within modecoupling theory (MCT), we discuss the threshold external force needed to delocalize the probe from a host glass, and its relation to strong nonlinear velocity-force curves in a host fluid. Experimental microrheology data and simulations, which we performed, are explained with a simplified model.
\end{abstract}

PACS numbers: 83.10.-y, 83.10.Rs, 64.70.pv

Microrheology is a promising technique providing local probes of the dynamics in a complex fluid 1]. Monitoring the motion of a singled-out probe particle embedded in a (dense) host fluid or gel, one addresses questions about the microscopic origins of the host's complex-fluid behavior and in particular the link between microscopic mechanisms and macroscopic properties amenable to conventional rheology. This connection subtly depends on the host, probe-bath interactions, and on the applied forces. Active microrheology turns this into an advantage, at the cost of requiring much better knowledge about the microscopic processes [2]: applying a known forcing to the individual particle, one explores the nonequilibrium and usually nonlinear regime, providing detailed insight into the structure-dynamics relationship, e.g., in cellular environments [3] or close to the glass transition [4, 5, 6]. Laser tweezers, magnetic or surface-chemistry forces [7] provide experimental realizations achieving large forcing.

The external-force-velocity relations obtained in dense suspensions reveal striking nonlinearities, induced by the slow relaxation of the host. Leaving the linear-response regime, a sudden strong increase in the velocity reveals the strength required to pull free the probe from the (transient) local neighbor cage, such that force-induced motion overrules structural relaxation. Recent theoretical progress [8, 9] notwithstanding, it remains to understand the nonlinear friction induced by the slow structural rearrangements of host particles.

Here we develop a theory for active nonlinear microrheology in suspensions close to their glass transition, establishing the conceptual connection between micro- and macro-rheology, when (de)localization of the probe occurs, and how the structure of the cage is distorted close to this yielding point. We start from microscopic equations of motion and relate the force-velocity relation of the probe, by virtue of an exact Green-Kubo-like formula, to a microscopic-force autocorrelation function. This can be approximated through non-equilibrium taggedparticle density correlation functions, which in turn are calculated in the framework of the mode-coupling theory of the glass transition (MCT) [10]. For a hard-sphere (HS) suspension with a pulled probe of same size as the host particles, we demonstrate that the theory predicts a delocalization threshold force that explains the nonlinear response seen in experiment and simulation.

We start from the many-body Smoluchowski equation for the nonequilibrium distribution function $\Psi(t)$ of a system of $N$ Brownian particles (positions $\boldsymbol{r}_{i}$ ) and a single probe (labeled $s), \partial_{t} \Psi(t)=\Omega \Psi(t)$. Subjecting only the probe particle to a constant, homogeneous force $\boldsymbol{F}^{\mathrm{ex}}$, the Smoluchowski operator $\Omega=\Omega_{0}+\Delta \Omega$ reads

$$
\Omega=\sum_{i=1, \ldots, N, s} \boldsymbol{\partial}_{i} \cdot\left(k_{B} T \boldsymbol{\partial}_{i}-\boldsymbol{F}_{i}\right) / \zeta_{i}-\left(\boldsymbol{\partial}_{s} \cdot \boldsymbol{F}^{\mathrm{ex}}\right) / \zeta_{s},
$$

where $\Delta \Omega=-\left(\boldsymbol{\partial}_{s} \cdot \boldsymbol{F}^{\mathrm{ex}}\right) / \zeta_{s}$ is the nonequilibrium term describing active forcing, and $\Omega_{0}$ the equilibrium timeevolution. We neglect solvent-induced hydrodynamic interactions and introduce Stokes friction coefficients for host $\left(\zeta_{i=1, \ldots N} \equiv \zeta_{0}\right)$ and probe $\left(\zeta_{s}\right)$ particles. The $\boldsymbol{F}_{i, s}$ are (potential) interaction forces among the particles.

To obtain nonequilibrium averages formed with the force-dependent Smoluchowski operator, the integrationthrough-transients (ITT) formalism [1] recasts Eq. (1):

$$
\Psi(t)=\Psi_{\mathrm{eq}}-\frac{k_{\mathrm{B}} T}{\zeta_{s}} \int_{0}^{t} d t^{\prime} \exp \left[\Omega t^{\prime}\right]\left(\boldsymbol{F}^{\mathrm{ex}} \cdot \boldsymbol{F}_{s}\right) \Psi_{\mathrm{eq}},
$$

assuming equilibrium at $t=0$. In particular, the stationary friction coefficient $\zeta\left(F^{\mathrm{ex}}\right)$, defined via the average stationary velocity at given external force

$$
\zeta\left\langle\boldsymbol{v}_{s}\right\rangle_{t \rightarrow \infty} \equiv \zeta\left\langle\boldsymbol{v}_{s}\right\rangle_{\infty}=\boldsymbol{F}^{\mathrm{ex}},
$$

is found by using Eq. (2) and equating external and interaction forces on the probe:

$$
\zeta=\zeta_{s}+\frac{1}{3 k_{\mathrm{B}} T} \int_{0}^{\infty} d t\left\langle\boldsymbol{F}_{s} \exp \left[\Omega^{\dagger}\left(\boldsymbol{F}^{\mathrm{ex}}\right) t\right] \boldsymbol{F}_{s}\right\rangle_{\mathrm{eq}} .
$$

This formally exact generalized Green-Kubo relation connects the far-from-equilibrium response to a transient 
equilibrium-averaged correlation function. ITT achieves that all following averages are equilibrium ones, denoted by $\langle\cdot\rangle$ (suppressing the eq subscript). $\Omega^{\dagger}$ is the adjoint of $\Omega$, and $\boldsymbol{F}^{\mathrm{ex}}$ enters non-perturbatively; linear response is recovered by neglecting this dependence.

Following MCT, we assume that force fluctuations are governed by collective and probe-particle density fluctuations, $\varrho_{\boldsymbol{q}}=\sum_{i=1}^{N} \exp \left[i \boldsymbol{q} \boldsymbol{r}_{i}\right]$ and $\varrho_{\boldsymbol{q}}^{s}=\exp \left[i \boldsymbol{q} \boldsymbol{r}_{s}\right]$. We take that in the thermodynamic limit, the motion of the probe has negligible impact on the bulk properties of the host, and restrict wave numbers to a discrete grid neglecting anomalous long distance correlations. Inserting a projector $\left.\mathcal{P}_{2} \propto \sum_{k p} \varrho_{k}^{s} \varrho_{p}\right\rangle\left\langle\varrho_{k}^{s} \varrho_{p}\right.$ on both sides of the operator exponential in Eq. (44), because forces on the probe relax by host particle rearrangements and probe motion, and splitting four-point density averages into dynamical density correlators, $\phi_{\boldsymbol{k}}(t)=\left\langle\varrho_{-\boldsymbol{k}} \exp \left[\Omega^{\dagger} t\right] \varrho_{\boldsymbol{k}}\right\rangle$ and $\phi_{\boldsymbol{k}}^{s}(t)=\left\langle\varrho_{-\boldsymbol{k}}^{s} \exp \left[\Omega^{\dagger} t\right] \varrho_{\boldsymbol{k}}^{s}\right\rangle$, we arrive at

$$
\left\langle\boldsymbol{F}_{s} \exp \left[\Omega^{\dagger} t\right] \boldsymbol{F}_{s}\right\rangle \approx \sum_{\boldsymbol{k}} \frac{\left|k_{\mathrm{B}} T k S_{k}^{s}\right|^{2}}{N S_{k}} \phi_{\boldsymbol{k}}^{s}(t) \phi_{-\boldsymbol{k}}(t) .
$$

$S_{k}=\left\langle\varrho_{\boldsymbol{k}} \varrho_{-\boldsymbol{k}}\right\rangle$ and $S_{k}^{s}=\left\langle\varrho_{\boldsymbol{k}}^{s} \varrho_{-\boldsymbol{k}}\right\rangle$ are the equilibrium structure functions describing interactions among probe and host particles.

The probe correlator $\phi_{\boldsymbol{q}}^{s}(t)$ is complex-valued, as the perturbed operator $\Omega^{\dagger}$ is non-Hermitian. This reflects that the probe-density distribution is shifted by application of an external force: while in equilibrium it is centered around the origin, the average position of the probe moves, introducing a complex-valued phase factor in $\phi_{\boldsymbol{q}}^{s}(t)$. Still, Eq. (5) maintains $\zeta \in \mathbb{R}$ due to the symmetry $\phi_{-\boldsymbol{q}}^{s}(t)=\left(\phi_{\boldsymbol{q}}^{s}(t)\right)^{*}$.

Equation (5) recasts the problem of calculating the probe friction as one of calculating collective and taggedparticle density correlation functions. To this end, we employ Zwanzig-Mori equations of motion [11],

$$
\partial_{t} \phi_{\boldsymbol{q}}^{s}(t)=-\omega_{\boldsymbol{q}, \boldsymbol{q}}^{s} \phi_{\boldsymbol{q}}^{s}(t)-\int_{0}^{t} d t^{\prime} m_{\boldsymbol{q}}^{s}\left(t-t^{\prime}\right) \partial_{t^{\prime}} \phi_{\boldsymbol{q}}^{s}\left(t^{\prime}\right)
$$

closed by the MCT approximation generalizing Eq. (5) to finite wave vectors,

$$
m_{\boldsymbol{q}}^{s}(t)=\frac{k_{\mathrm{B}} T}{\zeta_{s} \omega_{\boldsymbol{q}, \boldsymbol{q}}^{s}} \sum_{\boldsymbol{k}+\boldsymbol{p}=\boldsymbol{q}} \frac{1}{N S_{p}} \mathcal{V}_{\boldsymbol{q} \boldsymbol{k} \boldsymbol{p}}^{s} \mathcal{V}_{\boldsymbol{q} \boldsymbol{k} \boldsymbol{p}}^{s, \dagger} \phi_{\boldsymbol{k}}^{s}(t) \phi_{\boldsymbol{p}}(t)
$$

Again, the physical idea in the MCT approximation is that the friction kernel $m_{\boldsymbol{q}}^{s}(t)$ relaxes by both probe and host density dynamics. The coupling coefficients are $\mathcal{V}_{\boldsymbol{q} \boldsymbol{k} \boldsymbol{p}}^{s}=(\boldsymbol{q} \boldsymbol{p}) S_{p}^{s}, \mathcal{V}_{\boldsymbol{q} \boldsymbol{k} \boldsymbol{p}}^{s, \dagger}=\omega_{\boldsymbol{q}, \boldsymbol{p}}^{s} S_{p}^{s}$, where $\omega_{\boldsymbol{q}, \boldsymbol{p}}^{s}=$ $\left(\boldsymbol{q} k_{\mathrm{B}} T-i \boldsymbol{F}^{\mathrm{ex}}\right) \cdot \boldsymbol{p} / \zeta_{s}$. An analogous set of equations holds for $\phi_{\boldsymbol{q}}(t)$. Since the external force acts on the probe only, the $\phi_{\boldsymbol{q}}(t)$ are in fact determined by the unperturbed Smoluchowski operator, $\Omega_{0}$, resulting in the standard MCT scenario of glassy dynamics $[10,12]$. This describes arrest driven by wave vectors connected with a

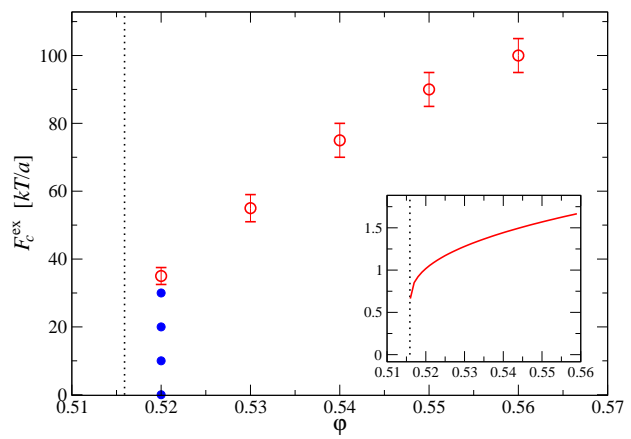

FIG. 1: Threshold force $F_{c}^{\mathrm{ex}}(\varphi)$ needed to delocalize a hardsphere probe particle in a glass of equally large hard spheres with packing fraction $\varphi$ above the glass transition (dotted line), calculated from MCT within the Percus-Yevick approximation. Blue circles mark $F^{\mathrm{ex}}$ values used in Fig. 2, Inset: corresponding schematic-model result (see text).

typical host particle radius $a$. Thus the dimensionless parameter measuring the effect of the external force is $a F^{\mathrm{ex}} /\left(k_{\mathrm{B}} T\right)$, the work required to pull the probe over that distance in relation to thermal energy.

The macroscopic counterpart to the friction $\zeta$ is the dispersion viscosity $\eta$ measured in bulk flow. Within ITT, the analog to Eq. (4) holds for the latter 13. MCT expresses this as a functional only of the host correlators $\phi_{\boldsymbol{q}}(t)$, while in Eq. (5), the probe correlators $\phi_{\boldsymbol{q}}^{s}(t)$ enter. In linear response close to the glass transition, identical scaling laws for both closely link micro- and macro-rheology [14]. For large external forces, this correspondence breaks: Equations (6) for the probe correlator contain a novel delocalization transition that is absent in $\phi_{\boldsymbol{q}}(t)$. A probe arrested in a glassy host suspension remains localized in its (deformed) nearest-neighbor cage (described by $f_{\boldsymbol{q}}^{s}=\phi_{\boldsymbol{q}}^{s}(t \rightarrow \infty)>0$ ), yielding zero average velocity (infinite friction) only below a finite threshold $F_{c}^{\mathrm{ex}}$. At larger force, the probe is pulled free $\left(f_{\boldsymbol{q}}^{s}=0\right)$ and attains a steady velocity (finite friction) at long times. In the liquid, cages are transient, and a remnant of the threshold survives as a sudden sharp "force thinning" in $\zeta\left(F^{\mathrm{ex}}\right)$.

The details of the delocalization transition depend on the host properties, which we model now as hard spheres using the known numerical MCT results for the collective density correlators $\phi_{\boldsymbol{q}}(t)$ within the Percus-Yevick

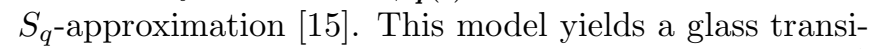
tion at packing fraction $\varphi_{c} \approx 0.516$, where $\varphi=(4 \pi / 3) \varrho a^{3}$ with number density $\varrho$ is the only parameter. Figure 1 shows our results for the delocalization threshold force, $F_{c}^{\text {ex }}$, for a probe equal to the host particles $\left(S_{q}=1+S_{q}^{s}\right)$. This threshold $F_{c}^{\mathrm{ex}}\left(\varphi_{c}\right)>0$ is finite at $\varphi_{c}$, and increases further with increasing density. Note that $F_{c}^{\mathrm{ex}}=\mathcal{O}\left(50 k_{\mathrm{B}} T / a\right)$, much larger than one might intuitively expect. This reflects the strong caging force exerted by the set of nearest neighbors that must be over- 


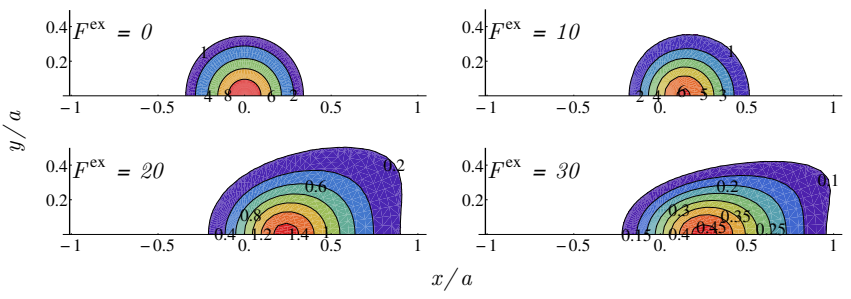

FIG. 2: Contour plot of the probability distribution $f^{s}(\boldsymbol{r})$ for a localized hard-sphere probe of radius $a$ in a hard-sphere system with same radius at $\varphi=0.52$, for external forces acting to the right with indicated magnitude in units of $k_{\mathrm{B}} T / a$.

come before the probe can be delocalized.

The inverse Fourier transform of $f_{\boldsymbol{q}}^{s}$ is the $t \rightarrow \infty$ probability distribution for the position of a probe starting at the origin; Figure 2 shows our results at packing fraction $\varphi=0.52$, slightly above the glass transition, for several forces below $F_{c}^{\mathrm{ex}} . \boldsymbol{F}^{\mathrm{ex}}$ is taken to be in (positive) horizontal direction, rendering $f^{s}(\boldsymbol{r})$ rotational-symmetric around this axis. For zero force, the distribution is spherical-symmetric and centered around the origin; it decays on a length scale of $0.2 a$, the typical localization length for solids dominated by hard-core repulsion. Small applied forces mainly shift the center of the distribution to a position $x_{0} \approx 0.2 a$, i.e., they push the probe to the "cage wall" without essentially distorting the cage. Close to the delocalization threshold, however, $f^{s}(\boldsymbol{r})$ develops a deformed tail extending into the force direction, reducing the spherical symmetry to a merely rotational one. Interestingly, the tail does not extend along the symmetry axis; rather, a "dip" is seen in direction of the applied force. For $F^{\mathrm{ex}} \geq F_{c}^{\mathrm{ex}}, f_{\boldsymbol{q}}^{s}$ and $f^{s}(\boldsymbol{r})$ vanish, indicating a delocalized state.

To discuss probe friction or similar dynamical quantities, we need to solve the time-dependent and spatially inhomogeneous Eqs. (6). A first step towards this is to solve a simplified, "schematic" MCT model. Take $\zeta=1+\int_{0}^{\infty} \phi^{s}(t) \phi(t) d t$, with

$$
\begin{gathered}
\partial_{t} \phi^{s}(t)+\omega^{s} \phi^{s}(t)+\int_{0}^{t} m^{s}\left(t-t^{\prime}\right) \partial_{t^{\prime}} \phi^{s}\left(t^{\prime}\right) d t^{\prime}=0 \\
m^{s}(t)=v_{s} \phi^{s *}(t) \phi(t)
\end{gathered}
$$

$\omega^{s}=1-i F^{\mathrm{ex}}$. The host correlator $\phi(t)$ is set by the " $\mathrm{F}_{12}$ model" often used to describe glassy dynamics in equilibrium [10, 16], governed by a separation parameter $\epsilon$ such that $\epsilon<0$ in the liquid, and $\epsilon \geq 0$ in the glass; $\epsilon$ thus measures the host interactions. $v_{s}$ describes the strength of probe-host coupling. Its $F^{\mathrm{ex}}$ dependence is ignored, neglecting the subtle interplay arising from static correlations involving more than one wave vector; still, the model recovers the qualitative behavior of the force threshold (see inset of Fig. 1). We expect the schematic model to be reasonable for small enough velocities and/or forces, where the friction is dominated by
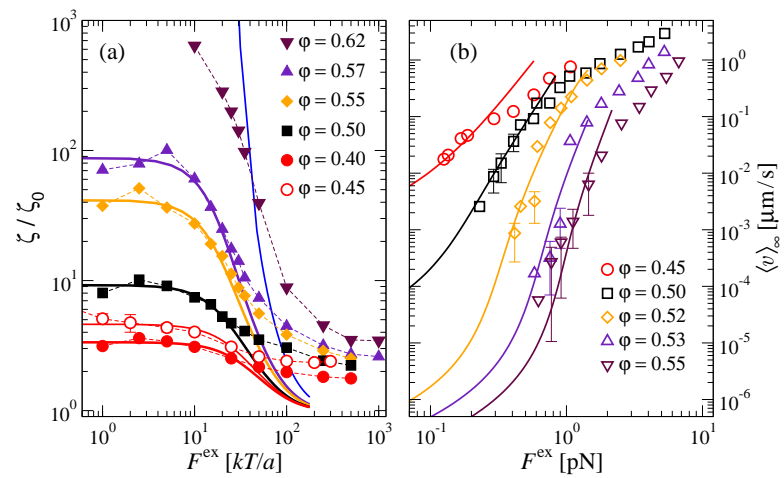

FIG. 3: (a) Probe friction $\zeta$ as a function of external force for packing fractions $\varphi$ as indicated, from simulations of a quasihard-sphere system (symbols), from Brownian dynamics for monodisperse HS (Ref. [17], open symbols), and from the schematic model (solid lines; see text) with $k_{\mathrm{B}} T / a=0.05586$, $1 / \zeta_{0}=3.44$, and parameters $\left(\epsilon, v_{s}\right)=(0,6),(-0.045,3.5)$, $(-0.058,3),(-0.18,2),(-0.5,1.5),(-0.8,1)$, top to bottom. (b) Open symbols: Experimental force-velocity relations for a colloidal suspension, from Ref. 5]. Lines: schematic model with $\left(\epsilon, v_{s}\right)=(-0.006,46),(-0.008,30),(-0.01,15)$, $(-0.05,6)$, and $v_{1}=v_{2}=0.1=v_{s} / 5$ (bottom to top), shifted along $x$ and $y$ by 0.058 and 296 .

universal features of the transition at $F_{c}^{\mathrm{ex}}$.

To test the simplified model, we performed simulations of a slightly polydisperse quasi-hard sphere system undergoing strongly damped Newtonian dynamics, which shows a glass transition at $\varphi_{c} \approx 0.595$ [18. Particles (mass $m=1, k_{\mathrm{B}} T=1$, radii distributed uniformly in $[0.9,1.1])$ suffer friction with the solvent $\left(\zeta_{0}=50\right)$ and random forces obeying the fluctuation-dissipation theorem. One particle is randomly selected to undergo an external force $\boldsymbol{F}^{\mathrm{ex}}$ until it reaches a distance half the size of the simulation box (elongated in the direction of $\boldsymbol{F}^{\mathrm{ex}}$ by a factor of 8 ). The average probe velocity is measured sampling more than 300 independent trajectories, and the friction is calculated using Eq. (3). All simulations were initially equilibrated, except for $\varphi=0.62$, where the system was aged for $t_{w}=25000$. At this density, results show little influence of ageing for forces $F^{\mathrm{ex}} \gtrsim 35 k_{\mathrm{B}} T / a$.

A strong decrease in the dynamical friction $\zeta$ around $F_{c}^{\mathrm{ex}}=\mathcal{O}\left(40 k_{\mathrm{B}} T / a\right)$ seen in the simulation [symbols in Fig. 3(a)] indicates the force threshold. Fitting $\epsilon$ and $v_{s}$ per curve, and two shift factors setting the units, the schematic model reproduces this behavior for $\varphi<\varphi_{c}$. In the (idealized) glass, it predicts a true delocalization transition as $\zeta \rightarrow \infty$ for $F^{\mathrm{ex}}<F_{c}^{\mathrm{ex}}$, exemplified by a $\epsilon=0$ curve in Fig. 3(a). In the simulation, $\zeta$ remains finite in the accessible window presumably because of ergodicity restoring processes ignored here [10]. Employing larger $v_{s}$, our model also explains recent experiments on colloidal systems using larger probes [5], as shown in Fig. 3(b). In these velocity-force curves obtained below the glass transition, the force-threshold signature is a 


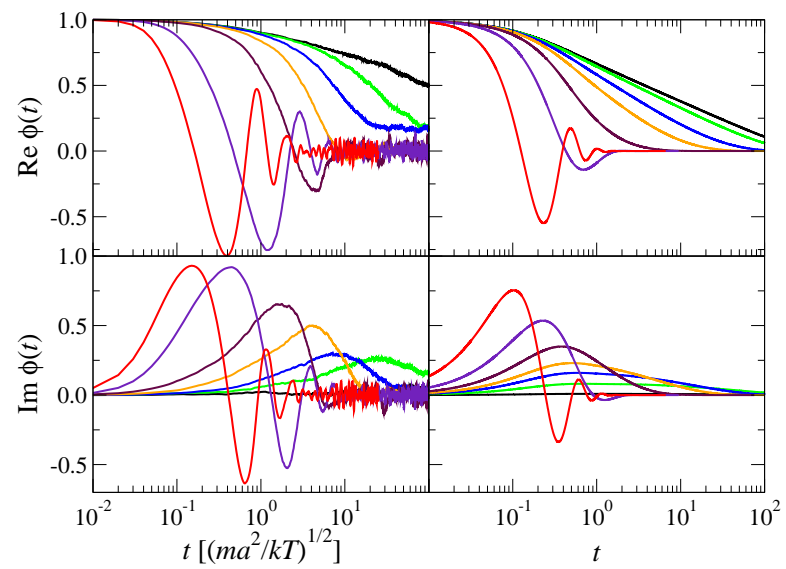

FIG. 4: Probe-particle density correlation function $\phi_{\boldsymbol{q}}^{s}(t)$ from computer simulation at $\varphi=0.55$ (left) and from schematic MCT (right; fit as in Fig. 3), real (top) and imaginary (bottom) parts. $\boldsymbol{q} \| \boldsymbol{F}^{\mathrm{ex}}$ corresponds to the position of the main peak in the static structure factor $S(q)$. For the simulation, $a F^{\mathrm{ex}} /\left(k_{\mathrm{B}} T\right)=1,10,20,30,50,100$, and 250 (right to left).

steep increase of $\left\langle\boldsymbol{v}_{s}\right\rangle_{\infty}$ around $F_{c}^{\mathrm{ex}} \approx 0.2 \mathrm{pN} \gg k_{\mathrm{B}} T / a$, again reproduced by the model. For too large external forces, the model fails, as expected above.

The virtue of the schematic model is to allow more detailed qualitative predictions for the slow nonequilibrium dynamics. This is demonstrated by Fig. 4, where we compare the tagged-particle density correlation function $\phi_{\boldsymbol{q}}^{s}(t)$ obtained from the simulation for a wave vector $\boldsymbol{q} \| \boldsymbol{F}^{\mathrm{ex}}$ with a magnitude corresponding to the nearestneighbor peak in $S_{q}$. The simulation confirms the existence of complex-valued correlation functions (for this $\boldsymbol{q}$-direction) as a signature of non-equilibrium, naturally arising in our microscopic framework. For $\boldsymbol{q}$ perpendicular to the external force, $\phi_{\boldsymbol{q}}^{s}(t)=\left(\phi_{-\boldsymbol{q}}^{s}(t)\right)^{*}$ remains realvalued, owing to the rotational symmetry $\phi_{\boldsymbol{q}}^{s}(t)=\phi_{-\boldsymbol{q}}^{s}(t)$, and exhibits the two-step decay typical for glass formers with an intermediate plateau and a final relaxation sped up by the external force. No such clear plateau is seen in the figure for $\boldsymbol{q}$ parallel to $\boldsymbol{F}^{\mathrm{ex}}$. For large forces, the $\phi_{\boldsymbol{q}}^{s}(t)$ show pronounced oscillations, quite unexpected for a Brownian system, and even stronger in the simulation data.

To summarize, we have developed a microscopically founded theory for the nonlinear active microrheology close to a glass transition. Starting from the Smoluchowski equation without hydrodynamic interactions, and applying approximations in the spirit of the modecoupling theory of the glass transition, we predict the probe friction as a function of the external force and of the equilibrium host structure.

The theory predics a finite microrheological force threshold needed to delocalize a probe from a glassy host, locally melting it. In the dense liquid, this is reflected by a strong nonlinear decrease in friction coefficients differentiating the regimes where cages are either broken by slow structural relaxation (for small external force), or by large enough applied force. A schematic model captures these aspects and allows to fit experimental and simulation data quantitatively for not too large external forces.

The force threshold could be related to the existence of a yield stress well established for glassy colloidal systems, and predicted by MCT for constant-velocity bulk driving. It will be promising to study more closely this relation and the dynamical behavior of the system close to microand macro-yielding.

We thank A. Erbe, W.C.K. Poon and J.F. Brady for discussions, and Deutsche Forschungsgemeinschaft (SFB 513 project B12), Helmholtz-Gemeinschaft (Hochschul-Nachwuchsgruppe VH-NG 406), M.E.C. project MAT-2006-13646-CO3-02 and Junta de Andalucía (P06-FQM-01869) for funding.

[1] T. A. Waigh, Rep. Prog. Phys. 68, 685 (2005).

[2] T. M. Squires, Langmuir 24, 1147 (2008).

[3] C. Wilhelm, Phys. Rev. Lett. 101, 028101 (2008).

[4] M. B. Hastings, C. J. Olson Reichhardt, and C. Reichhardt, Phys. Rev. Lett. 90, 098302 (2003).

[5] P. Habdas, D. Schaar, A. C. Levitt, and E. R. Weeks, Europhys. Lett. 67, 477 (2004).

[6] S. R. Williams and D. J. Evans, Phys. Rev. Lett. 96, 015701 (2006).

[7] L. Baraban, A. Erbe, P. Leiderer, and P. Kühler, submitted to Phys. Rev. Lett., arXiv:0807.1619.

[8] T. M. Squires and J. F. Brady, Phys. Fl. 17, 073101 (2005).

[9] R. L. Jack, D. Kelsey, J. Garrahan, and D. Chandler, Phys. Rev. E 78, 011506 (2008).

[10] W. Götze and L. Sjögren, Rep. Prog. Phys. 55, 241 (1992).

[11] M. Fuchs and M. E. Cates, Phys. Rev. Lett. 89, 248304 (2002).

[12] W. van Megen and S. M. Underwood, Phys. Rev. Lett. 70, 2766 (1993).

[13] J. M. Brader, Th. Voigtmann, M. E. Cates, and M. Fuchs, Phys. Rev. Lett. 98, 058301 (2007).

[14] M. Fuchs and M. R. Mayr, Phys. Rev. E 60, 5742 (1999), and references therein.

[15] T. Franosch, M. Fuchs, W. Götze, M. R. Mayr, and A. P. Singh, Phys. Rev. E 55, 7153 (1997).

[16] We use Eq. (7) with $\omega=1$ for the correlator $\phi(t)$ and memory kernel $m(t)=v_{1} \phi(t)+v_{2} \phi(t)^{2}$ with $v_{1,2}=$ $v_{1,2}^{c}(1+\epsilon)$ and $\left(v_{1}^{c}, v_{2}^{c}\right)=(0.828 \ldots, 2)$.

[17] I. C. Carpen and J. F. Brady, J. Rheol. 49, 1483 (2005).

[18] Th. Voigtmann, A. M. Puertas, and M. Fuchs, Phys. Rev. E 70, 061506 (2004). 